\title{
Intrapartum Fetal Heart Monitoring Practices in Selected Facilities in Aspirational Districts of Jharkhand, Odisha and Uttarakhand
}

\author{
Enisha Sarin ${ }^{1}$ (D) Devina Bajpayee ${ }^{2} \cdot$ Arvind Kumar $^{3} \cdot$ Sourav Ghosh Dastidar ${ }^{4} \cdot$ Subodh Chandra $^{5} \cdot$ Ranjan Panda $^{6,7}$. \\ Gunjan Taneja ${ }^{3} \cdot$ Sachin Gupta ${ }^{3,8} \cdot$ Harish Kumar $^{3}$
}

Received: 11 May 2020 / Accepted: 20 November 2020 / Published online: 17 January 2021

(c) The Author(s) 2021

\begin{abstract}
Background The risk of mortality for the mother and the newborn is aggravated during birth in low- and middle-income countries due to preventable causes, which can be addressed with increased quality of care practices. One such practice is intrapartum fetal heart rate (FHR) monitoring, which is crucial for the early detection of fetal ischemia, but is inadequately monitored in low- and middle-income countries. In India, there is currently a lack of sufficient data on FHR monitoring.

Methods An assessment using facility records, interviews and observation was conducted in seven facilities providing tertiary, secondary or primary level care in aspirational districts of three states. The study sought to investigate the frequency of monitoring, devices used for monitoring and challenges in usage.

Results FHR was not monitored as per standard protocol. Case sheets revealed $70 \%$ of labor was monitored at least once. Only 33\% of observed cases were monitored every half hour during active labor, and none were monitored every $5 \mathrm{~min}$ during the second stage of labor. More time was observed for monitoring with a Doppler compared with a stethoscope, as providers reported fluctuation in readings. Reportedly, low audibility and a perceived need of expertise were associated with using a stethoscope. High case load and the time required for monitoring were reported as challenges in adhering to standard monitoring protocols.

Conclusion The introduction of a standardized device and a short refresher training on the World Health Organization and skilled birth attendant protocols for FHR monitoring will improve usage and compliance.
\end{abstract}

Keywords Fetal heart rate monitoring $\cdot$ Maternal and neonatal health $\cdot$ Fetal ischemia $\cdot$ India $\cdot$ Doppler

Enisha Sarin: Senior Advisor, Monitoring Evaluation and Learning, USAID-VRIDDHI/IPE Global, New Delhi, India, Devina Bajpayee: Senior Advisor, Maternal and Newborn Health, USAID-VRIDDHI/IPE Global, New Delhi, India, Arvind Kumar: National Data Manager, USAID-VRIDDHI/IPE Global, New Delhi, India, Sourav Ghosh Dastidar: State Technical Officer, Jharkhand, USAID-VRIDDHI/IPE Global, Ranchi, India, Subodh Chandra: State Technical Officer, Uttarakhand, USAID-VRIDDHI/ IPE Global, Dehradun, India, Office of Director General Medical, Health and Family Welfare, Dehradun, India Ranjan Panda: State Technical Advisor, Odisha, USAID-VRIDDHI/IPE Global, Bhubaneswar, India, Gunjan Taneja: USAID-VRIDDHI/IPE Global, New Delhi, India, Sachin Gupta: Advisor - Maternal \& Child Health, USAID, New Delhi, India, American Embassy, New Delhi, India, Harish Kumar: USAID-VRIDDHI/IPE Global, New Delhi, India.

Enisha Sarin esarin@ipeglobal.com

Extended author information available on the last page of the article

\section{Introduction}

Childbirth is a normal physiological process; however, in low- and middle-income countries (LMICs) there is an increased risk of mortality for the mother and her newborn at the time of birth, due to preventable causes. Over onethird of maternal deaths and life-threatening conditions $[1,2]$, approximately half of all stillbirths and a quarter of neonatal deaths result from complications during labor and childbirth [3].

India witnesses 32,000 maternal and 640,000 newborn deaths every year $[4,5]$, and while most of these occur during the period around birth, efforts to improve access to care have not resulted in a concomitant decline in mortality [6], severely calling into question the quality of care provided. Evidence also suggests other persistent causes, such as heavy workloads, which limit the time for history taking, thorough assessments and the provision of timely care; inadequate 
pre-service and in-service training of health staff; lack of awareness of current recommended effective practices and the availability of skilled personnel; lack of compliance to standard operating procedures; overcrowding at the point of care; and lack of drugs, supplies and equipment to provide effective care [7].

Intrapartum fetal heart rate (FHR) monitoring is crucial for the early detection of fetal ischemia. Prolonged intrapartum fetal ischemia often results in fresh stillbirth or a severely asphyxiated neonate. However, lack of adequate monitoring of FHR is a hallmark of many resource poor settings $[8,9]$. The World Health Organization (WHO) recommends listening to the fetal heart rate immediately after a contraction and counting the fetal heart rate for a full minutes at least once every $30 \mathrm{~min}$ during the active phase of the first stage of labor and every 5 min during the second stage [10].

A variety of tools and methods used for FHR monitoring in LMIC include stethoscope, Pinard fetal stethoscope, handheld Doppler device or a cardiotocogram (CTG). A Cochrane review concluded that while the use of intermittent CTG and handheld Doppler led to a higher likelihood of the detection of FHR and increased the likelihood of cesarean section for fetal distress compared to a Pinard, there was no difference in perinatal mortality [11]. Pregnant women prefer a hands-on approach to care and are likely to favor any technique that allows this. Service providers prefer to use a Doppler because it offers reassurance and potentially leads to better outcomes for women [12].

Given the inadequate status of monitoring of FHR and the absence of the literature around it in the Indian public health system, the United States Agency for International Development (USAID) Vriddhi project undertook an assessment to understand the current situation of FHR monitoring across different levels of public health facilities.

\section{Methods}

\section{Study Design and Objectives}

A mixed methods study, using record review, key informant interviews and observation of labor, was conducted to assess.

- Frequency of FHR monitoring

- Detection level of abnormal FHR

- Devices used and challenges in usage

\section{Selection of Facilities}

The Vriddhi project provides technical support to the Government of India's LaQshya Labor Room Quality
Improvement Initiative (Table 1). Three aspirational districts from the states of Jharkhand, Odisha and Uttarakhand were purposively selected for the assessment. A total of seven facilities, three community health centers (CHC), three district hospitals (DH) and a medical college (MC), were assessed on practices and operational challenges. These levels were selected as CHC are the first referral point from a primary healthcare center, district hospitals are the final referral centers for the primary and secondary levels of public health system, and a medical college provides specialized care.

\section{Selection of Study Participants}

Thirty-four service providers per facility was selected purposively for interviews. Thirty-four labor cases per facility were conveniently selected for observation based on the willingness of the pregnant woman and the service provider to participate. Permission was obtained from the National Health Mission, state officials and hospital authorities. Informed consent was obtained from providers and patients before proceeding with observation and interviews. In total, 26 service providers were interviewed, and 27 labor cases were observed. Data collection covered the period from March to June 2019 and was conducted by Vriddhi state team members.

\section{Tools}

Case sheets and partographs (wherever available) were reviewed by Vriddhi team for monitoring frequency, detection of abnormal FHR and actions taken. The interview questionnaire and the observation checklist, developed by project team, captured the type of device used to monitor FHR, challenges encountered in their use, and whether the staff knowledge staff and the monitoring practice was consistent with WHO standards.

\section{Data Analysis}

The data from records were entered in the project web portal. Previous 4-month data were reviewed for all but two facilities in Odisha, which had collected data for 3 months. The data were compiled in a separate Excel sheet and tabulated for simple frequency and percentages at an aggregate level and facility level. Interview and observation data were likewise entered on Excel sheet.

\section{Ethical Consideration}

The study used data that were publicly available in facility records, and therefore, no ethical clearance was required. Permission was obtained from the Ministry of Health and 
Table 1 Facilities across states

\begin{tabular}{llll}
\hline State & District hospital & $\begin{array}{l}\text { Community } \\
\text { health center } \\
\text { (CHC) }\end{array}$ & Medical college \\
\hline Jharkhand & Chaibasa & Ratu & $\begin{array}{c}\text { Rajendra Institute } \\
\text { of Medical Sci- } \\
\text { ences (RIMS) }\end{array}$ \\
$\begin{array}{l}\text { Odisha } \\
\text { Uttarakhand }\end{array}$ & $\begin{array}{l}\text { Kandhamal } \\
\text { Haridwar }\end{array}$ & $\begin{array}{l}\text { Baliguda } \\
\text { Manglore }\end{array}$ & \\
\hline
\end{tabular}

Table 2 Total frequency of monitoring of FHR according to record review $(\mathrm{N}=7310)$

\begin{tabular}{lll}
\hline No of cases never monitored & 559 & $7.6 \%$ \\
No of cases monitored once & 2330 & $32 \%$ \\
No of cases monitored twice & 771 & $10.6 \%$ \\
No of cases monitored 3-4 times & 898 & $12.3 \%$ \\
No of cases monitored more than 4 times & 1124 & $15.4 \%$ \\
No records available & 1628 & $22.3 \%$ \\
\hline
\end{tabular}

Family Welfare, the respective state health officials and the facility in charge. Provider interviews and observation of labor cases were conducted only after obtaining written consent from both providers and pregnant women.

\section{Results}

Stethoscope and Doppler were commonly used. CTG was available in the medical college and in two of the district hospitals and used only in a few cases.

\section{Frequency of Monitoring}

Record review highlights that while FHR was monitored at least once in a majority of labor cases (70 percent), it was not frequently monitored after that. About a quarter (27\%) were monitored three time and more (Table 2). Due to missing reports, there may be cases where FHR was monitored, but not recorded. The medical college conducted the least amount of monitoring. This may be due to the practice being performed, but not documented because of the very high case load. On observation, it was found that the four cases observed were monitored at 1-1 1/2 hours; however, documentation was only for time of admission.

For interviews and observation, monitoring was measured using the standard WHO guidelines for the first and second stages of labor. The table below illustrates this:

A total of 27 labor cases were observed. The observation period varied, lasting from one hour to five hours. Observation was done across stage $1(n=14)$ and stage 2 $(n=6)$ labor. Seven additional labor cases were observed across both the stages. A total of 26 service providers were interviewed with questions pertaining to both stages 1 and 2 of labor.

While $69 \%$ responded that they monitored FHR every half an hour in stage 1, during observation, only $33 \%$ monitored in that frequency (Table 3 ). There was no reporting or observation of frequent monitoring for the second stage of labor. Observers also examined the case sheets. Many cases were monitored outside the range of standard frequency.

Medical college had the lowest percentage (1\%) of cases never monitored compared to the other facilities, reflecting that almost every case is monitored. The frequency of FHR monitoring did not account for the time between admission and delivery. Pregnant women access medical colleges from far and often reach in advanced stage of labor. The frequency of FHR monitoring is consequently low. Only one CHC followed correct monitoring for stage 1 (Table 3).

\section{Reasons for not Complying with Standard Protocol}

Reasons given for not monitoring according to standard protocols included high case load $(n=16)$; too few staff to monitor as required by the guidelines $(n=15)$; and taking too much time $(n=5)$.
Table 3 Frequency of monitoring FHR as per standard guideline (interview and observation data)

\begin{tabular}{|c|c|c|c|c|c|c|c|}
\hline Stages of labor & $\begin{array}{l}\text { Once } \\
\text { every } \\
\text { hour }\end{array}$ & $\begin{array}{l}\text { Once } \\
\text { every } \\
30 \text { min }\end{array}$ & $\begin{array}{l}\text { Once } \\
\text { every } \\
15 \text { min }\end{array}$ & $\begin{array}{l}\text { Once } \\
\text { every } \\
5 \text { min }\end{array}$ & $\begin{array}{l}\text { Only at } \\
\text { admission }\end{array}$ & Any other & Missing \\
\hline \multicolumn{8}{|l|}{ Interview $(N=26)^{*}$} \\
\hline Stage 1 & 4 & 18 & & & & 4 & \\
\hline Stage 2 & & 3 & 8 & 1 & & & \\
\hline \multicolumn{8}{|l|}{ Observation $(N=27)$} \\
\hline Stage $1\left(\mathrm{n}=14+7^{* *}\right)$ & 2 & 7 & 0 & 0 & 4 & 7 & 1 \\
\hline $\begin{array}{l}\text { Stage } 2 \\
\left(\mathrm{n}=6+7^{* *}\right)\end{array}$ & & 4 & 2 & 0 & 2 & 4 & 1 \\
\hline
\end{tabular}

*Interview questions have multiple choice responses, so frequencies do not add up to $\mathrm{N}$

** An additional 7 observers observed both stage 1 and stage 2 


\section{Detection of Abnormal FHR}

Out of 7310 deliveries, 254 abnormal FHR cases (3.5\%) were detected. The majority (63\%) of abnormal FHR cases underwent $\mathrm{C}$-section, and $13 \%$ were referred out (Table 4 ). $\mathrm{C}$-sections comprised $22 \%$ of the total cases with abnormal FHR responsible for almost $10 \%$ of these. While the total still birth and asphyxia rate was $2 \%$ and $6 \%$, respectively, the contribution of abnormal FHR to the cases was not recorded.

The tertiary facility performed the largest share of C-sections among all facilities, followed by district hospitals. CHCs, on the other hand, referred $25 \%$ of abnormal FHR cases. Assisted delivery was low at all levels.

\section{Use and Reliability of Devices Used}

Nearly $77 \%$ of the providers reported using a stethoscope, and $58 \%$ did so in combination with a handheld Doppler. On observation, an equal number of labor cases were monitored through either a stethoscope or a Doppler (Table 5). The use of CTG was not observed. Interview and observation data show a higher average time to take Doppler readings in contrast to stethoscope readings. Individual readings of different devices could not be separated out when a combination of devices was used.

Providers reported difficulty measuring FHR with both stethoscopes and with handheld Doppler. One difficulty related to stethoscope was the time needed to locate a heartbeat. This is contrary to observation findings, which suggests this difficulty is merely a matter of the healthcare staff's perception. Limited audibility of stethoscope and expertise to use it were other challenges. For Doppler, challenges included difficulty locating the FHR site, fluctuating readings, insufficient numbers as per the total number of daily labor cases, and issues of battery replacement and storage. The Dopplers used in the facilities were of various makes, indicating that there was no one standardized measure in use. With the exception of the district hospital in Odisha, the facilities procured Dopplers locally.

\section{Discussion}

Monitoring did not adhere to standard guidelines. The majority of providers reported monitoring every $30 \mathrm{~min}$; on observation, this standard practice was only seen in 33 percent of the labor cases. This may be due to a social acceptability bias where, during interviews, providers overestimate the frequency of monitoring.

Almost a third of the cases were reported and observed to have been monitored only once at admission and at no other time. Some of the women came to the facility at an advanced stage of labor or immediately before birth, and therefore, there was only time to monitor FHR once. Nevertheless, these frequencies are comparable to those found in a study in Tanzania [12]. In that study, baseline rates of monitoring were extremely low, but, after the implementation of Doppler, monitoring of $<30$ min rose to 13 percent of the cases and monitoring between $30-60$ min rose to 38 percent, rates already seen in our study. This is perhaps due

Table 4 Facility level detection of abnormal FHR and action taken

\begin{tabular}{lllllll}
\hline Facility level & Total delivery & Total C-section N(\%*) & $\begin{array}{l}\text { Total detection of } \\
\text { abnormal FHR }\end{array}$ & $\begin{array}{l}\text { C-section of } \\
\text { abnormal FHR } \\
\text { cases }\end{array}$ & $\begin{array}{l}\text { Referrals of } \\
\text { abnormal FHR } \\
\text { cases }\end{array}$ & $\begin{array}{l}\text { Assisted delivery of } \\
\text { abnormal FHR cases }\end{array}$ \\
\hline District hospital & 3291 & $424(13 \%)$ & $159(5 \%)$ & $87(55 \%)$ & $18(11 \%)$ & $6(4 \%)$ \\
CHC & 1786 & $225(13 \%)$ & $60(3 \%)$ & $22(37 \%)$ & $15(25 \%)$ & 0 \\
Medical college & 2233 & $976(44 \%)$ & $75(3 \%)$ & $51(68 \%)$ & 0 & $1(1 \%)$ \\
\hline
\end{tabular}

*Percentages are rounded

Table 5 Types of device used at facility levels and average time taken for one reading*

\begin{tabular}{|c|c|c|c|c|c|c|}
\hline \multirow[t]{2}{*}{ Device } & \multicolumn{2}{|l|}{ Medical college } & \multicolumn{2}{|l|}{ District hospital } & \multicolumn{2}{|l|}{$\mathrm{CHC}$} \\
\hline & $\begin{array}{l}\text { Observation } N \\
\text { (time taken) }\end{array}$ & $\begin{array}{l}\text { Interview } N \\
\text { (time taken) }\end{array}$ & $\begin{array}{l}\text { Observation } N \\
\text { (time taken) }\end{array}$ & $\begin{array}{l}\text { Interview } N \\
\text { (time taken) }\end{array}$ & $\begin{array}{l}\text { Observation } N \\
\text { (time taken) }\end{array}$ & $\begin{array}{l}\text { Interview } N \\
\text { (time taken) }\end{array}$ \\
\hline $\begin{array}{l}\text { Stethoscope } \\
\text { (time } \\
\text { taken) }\end{array}$ & $4(2.5 \mathrm{~min})$ & & $5(1.5 \mathrm{~min})$ & $4(2 \min )$ & $3(1.5 \mathrm{~min})$ & $2(1.5 \mathrm{~min})$ \\
\hline Doppler & & & $6(3.5 \mathrm{~min})$ & $1(7 \min )$ & 7 (4.1 min) & $5(6.5 \min )$ \\
\hline CTG & & & $1(5 \min )$ & & & \\
\hline
\end{tabular}

*Table does not show combinations of devices 
to the Government of India's renewed focus on care around birth and the introduction of Doppler in many public health settings in India, which resulted in improved monitoring of FHR. However, the rates in the study still fall far below standard monitoring protocols. Providers maintained that they could not rigorously follow the guidelines due to the high ratio of case load to the number of available staff, a finding echoed in another study [13].

Abnormal FHR was detected in 3-5\% of total deliveries in the facilities, slightly higher than what was seen in an African study, where the detection rate of abnormal FHR was 1 percent using a Pinard [12]. In general, in middleincome countries, the detection of abnormal FHR with a Doppler is found to be higher compared to a Pinard [12, 14, 15]. Although health providers reported problems like time needed and low audibility of the stethoscopes, they used them in conjunction with a Doppler.

$\mathrm{C}$-sections were performed on a majority of the cases detected with abnormal FHR. The C-section rate was lower in CHC than in district hospitals and the medical college, while referral was relatively higher, indicating that they need to improve emergency obstetric care. Only one CHC in Jharkhand had the ability perform C-sections. Assisted delivery was low at all levels, as most of the cases ended up delivering by $\mathrm{C}$-section. Abnormal FHR was responsible for $10 \%$ of the total C-sections; thus, the additional C-sections could have been elective or performed for other obstetric complications.

Fresh stillbirth rate in the study was two percent of total deliveries. However, it was not documented how many of these were the result of abnormal FHR. There is currently no evidence on positive neonatal outcomes by using one or the other devices [16].

Providers found the Doppler easier to use, but there were issues around locating the site of the FHR, the reliability of the readings, and batteries and storage. Fewer Dopplers were available than required for the case load, resulting in providers relying on stethoscopes. However, the stethoscope had its own problems, as it requires expertise to use. There were also issues around audibility. An interesting point is that while providers believed a stethoscope takes time to auscultate, on observation, they only needed a minute and a half, suggesting their actual proficiency in using the stethoscope. Many of the providers were also seen to fall back on the stethoscope. Perhaps they found it easier to listen to the heartbeat after getting fluctuating readings on the Doppler. Local purchases of Dopplers are not regulated, and hence, there is no guarantee of quality control. It is possible that the use of non-standardized devices made readings confusing and unreliable. In other settings, the Doppler is found to provide reliable readings and thus is well accepted by providers [15, 17]. Indeed, it has been found that handheld devices, including stethoscopes, provide a more hands-on, woman-centered approach to care that is well accepted by both providers and patients [17].

\section{Limitations}

Due to limitations in documentation common in public health facilities, the record review suffers from missing data. It was not possible to exclude pregnant women who presented with full cervical dilatation from the analysis, so cases that required frequent monitoring could not be separated from those that did not. Additionally, the cases of abnormal FHR were not examined individually to detect what specific actions were taken. Results cannot be generalized, as facilities were purposively selected in just three states. However, the district hospital data, $\mathrm{CHC}$ data and medical college data can be reasonably seen as representative. Furthermore, observation was not conducted for the entire period of labor, but captured only a segment of the labor. Observation was supplemented by record review preceding and following the observation stage.

\section{Conclusions}

Monitoring of FHR is not performed at the desired frequency in accordance with standard guidelines. This may lead to poor neonatal outcomes, necessitating an intervention to improve compliance. Providers in the study reported challenges in using Dopplers, which may be due to the use of non-standardized equipment. Providers also reported challenges in the use of stethoscopes. The introduction of a standard Doppler which is globally recognized and approved could improve usage and compliance to protocol. Simultaneously, it needs to be tested in order to generate learning. The introduction of such a device is feasible within the given environment of focused intrapartum care. Alongside, a short refresher training or orientation on the WHO and SBA protocol for FHR monitoring would help to reorient labor room staff on the importance and need of frequent FHR monitoring.

Acknowledgements We wish to acknowledge the support of the state governments of Jharkhand, Odisha and Uttarakhand. We are also grateful to the project state and district consultants for collection of data.

Author contributions ES analyzed and interpreted the data, developed tools, and wrote the first and final draft of the manuscript, DB helped conceptualize the assessment and provided valuable inputs to the manuscript, SC and RP supported acquiring the data and provided inputs into results, GT critically reviewed the manuscript and helped edit it, SG provided critical review and HK contributed to conceptualizing and reviewing the work. 
Funding This work was made possible by the support of the American people through the United States Agency for International Development and its VRIDDHI (scaling up RMNCH + A Interventions) Project, implemented by IPE Global Ltd under the terms of Cooperative Agreement Number AID 386 A 14 00001. The contents of this paper represent the views of the authors and do not necessarily reflect the views of USAID.

Data Availability The datasets used and analyzed during the current study are available from the corresponding author on reasonable request.

\section{Compliance with Ethical Standards}

Conflict of interest The authors declare that they have no conflict of interest.

Ethics Approval The study used routine facility data for review with due permission from state health authorities and hospital/facility authorities. These are data that are collected as a routine part of documentation and fall under the category of publicly available information and hence waived from ethical consent (Indian Council of Medical Research, Ethical guidelines for biomedical research on human participants. Indian Council of Medical Research, 2006). Voluntary informed consent was obtained from service providers as well as pregnant women before conducting the interviews and observing labor. Anonymity and patients'/clinicians' rights were respected. The procedures used in this study adhere to the tenets of the Declaration of Helsinki.

Open Access This article is licensed under a Creative Commons Attribution 4.0 International License, which permits use, sharing, adaptation, distribution and reproduction in any medium or format, as long as you give appropriate credit to the original author(s) and the source, provide a link to the Creative Commons licence, and indicate if changes were made. The images or other third party material in this article are included in the article's Creative Commons licence, unless indicated otherwise in a credit line to the material. If material is not included in the article's Creative Commons licence and your intended use is not permitted by statutory regulation or exceeds the permitted use, you will need to obtain permission directly from the copyright holder. To view a copy of this licence, visit http://creativecommons.org/licenses/by/4.0/.

\section{References}

1. Kassebaum NJ, Bertozzi-Villa A, Coggeshall MS, Shackelford KA, Steiner C, Heuton KR, et al. Global, regional, and national levels and causes of maternal mortality during 1990-2013: a systematic analysis for the Global Burden of Disease Study 2013. Lancet. 2014;384(9947):980-1004.

2. Say L, Chou D, Gemmill A, Tunçalp Ö, Moller AB, Daniels J, et al. Global causes of maternal death: a WHO systematic analysis. Lancet Glob Health. 2014;2(6):e323-33.

3. Lawn JE, Blencowe H, Waiswa P, Amouzou A, Mathers C, Hogan D, et al. Stillbirths: rates, risk factors, and acceleration towards 2030. Lancet. 2016;387(10018):587-603.

4. WHO/ India has achieved groundbreaking success in reducing maternal mortality: World Health Organization; 2018 (http://www.searo.who.int/mediacentre/features/2018/india -groundbreaking-sucess-reducing-maternal-mortality-rate/en/) Accessed 12 May 2019
5. Million Death Study Collaborators. Changes in cause-specific neonatal and 1-59-month child mortality in India from 2000 to 2015: a nationally representative survey. Lancet 2017;390

6. Chaturvedi S, Randive B, Diwan V, De Costa A. Quality of obstetric referral services in India's JSY cash transfer programme for institutional births: a study from Madhya Pradesh province. PLoS ONE. 2014;9(5):e96773. https://doi. org/10.1371/journal.pone.0096773.

7. Munabi-Babigumira S, Glenton C, Lewin S, Fretheim A, Nabudere $\mathrm{H}$. Factors that influence the provision of intrapartum and postnatal care by skilled birth attendants in low- and middleincome countries: a qualitative evidence synthesis. Cochrane Database Syst Rev. 2017. https://doi.org/10.1002/14651858. CD011558.pub2.

8. Kc A, Wrammert J, Clark RB, Ewald U, Målqvist M. Inadequate fetal heart rate monitoring and poor use of partogram associated with intrapartum stillbirth: a case-referent study in Nepal. BMC Pregnancy Childbirth. 2016. https://doi.org/10.1186/ s12884-016-0806-2.

9. Yisma E, Dessalegn B, Astatkie A, Fesseha N. Completion of the modified World Health Organization (WHO) partograph during labor in public health institutions of Addis Ababa Ethiopia. Reprod Health. BioMed Central. 2013;10:23. https://doi. org/10.1186/1742-4755-10-23.

10. WHO Reproductive Health Library. WHO recommendation on intermittent fetal heart rate auscultation during labour. February 2018. The WHO Reproductive Health Library; Geneva.

11. Martis R, Emilia O, Nurdiati DS, Brown J. Intermittent auscultation (IA) of fetal heart rate in labour for fetal wellbeing. Cochrane Database Syst Rev. 2017. https://doi. org/10.1002/14651858.CD008680.pub2.

12. Kamala BA, Ersdal HL, Dalen I, Abeid MS, Ngarina MM, Perlman JM, et al. Implementation of a novel continuous fetal Doppler (Moyo) improves quality of intrapartum fetal heart rate monitoring in a resource-limited tertiary hospital in Tanzania: an observational study. PLoS ONE. 2018;13(10):e0205698. https://doi.org/10.1371/journal.pone.0205698.

13. Nyamtema AS, Urassa DP, Massawe S, Massawe A, Lindmark G, Van Roosmalen J. Staffing needs for quality perinatal care in Tanzania. Afr J Reprod Health. 2008;12:113-24.

14. Byaruhanga R, Bassani DG, Jagau A, et al. Use of wind-up fetal Doppler versus Pinard for fetal heart rate intermittent monitoring in labour: a randomized clinical trial. BMJ Open. 2015;5:e006867. https://doi.org/10.1136/bmjopen-2014-00686 7.

15. Mahomed K, Nyoni R, Mulambo T, et al. Randomised controlled trial of intrapartum fetal heart rate monitoring. BMJ. 1994;308:497-500.

16. Blix E, Maude R, Hals E, Kisa S, Karlsen E, Nohr EA, et al. Intermittent auscultation fetal monitoring during labour: a systematic scoping review to identify methods, effects, and accuracy. PLoS ONE. 2019. https://doi.org/10.1371/journ al.pone.0219573.

17. Downe S, Lawrie TA, Finlayson K, Oladapo OT. Effectiveness of respectful care policies for women using routine intrapartum care services: a systematic review. Reprod Health. 2018;15:23.

Publisher's Note Springer Nature remains neutral with regard to jurisdictional claims in published maps and institutional affiliations. 


\section{About the Author}

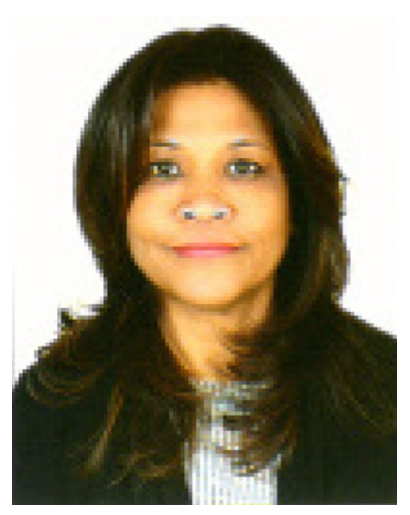

Dr. Enisha Sarin has been a public health researcher for twenty-five years working in the area of HIV prevention initially and currently in maternal and child health. Her focus areas have been wide-ranging, grounded in the social and behavioral aspects of healthhealthcare utilization among marginalized populations, health systems strengthening, gender disparity in health and quality of care in reproductive, maternal, neonatal and child health. Her expertise in both qualitative and quantitative research complements program implementation through the execution of timely and periodic evaluation of programs. Dr Sarin has a masters in Clinical Psychology from Delhi University and completed her masters, doctoral and postdoctoral program from the Johns Hopkins University Bloomberg School of Public Health, Department of International Health.

\section{Affiliations}

\section{Enisha Sarin ${ }^{1}\left[\right.$. Devina Bajpayee ${ }^{2} \cdot$ Arvind Kumar $^{3} \cdot$ Sourav Ghosh Dastidar ${ }^{4} \cdot$ Subodh Chandra $^{5} \cdot$ Ranjan Panda $^{6,7}$. Gunjan Taneja ${ }^{3}$. Sachin Gupta ${ }^{3,8} \cdot$ Harish Kumar ${ }^{3}$}

1 Monitoring Evaluation and Learning, USAID-VRIDDHI/IPE Global, B-84, Defence Colony, New Delhi 110024, India

2 Maternal and Newborn Health, USAID-VRIDDHI/IPE Global, B-84, Defence Colony, New Delhi 110024, India

3 USAID-VRIDDHI/IPE Global, B-84, Defence Colony, New Delhi 110024, India

4 USAID-VRIDDHI/IPE Global, GVI Campus, RCH Namkum, Ranchi, Jharkhand, India
5 Office of Director General Medical, Health and Family Welfare, Danda Lakhond, Post- Gujrada, Shasrdhara Road, Dehradun, Uttarakhand 248001, India

6 USAID-VRIDDHI/IPE Global, Bhubaneswar, India

7 Bhubaneswar, India

8 USAID, American Embassy, Shantipath, Chanakyapuri, New Delhi 110021, India 